\title{
Effects of Chinese herbal formula Erxian decoction for treating osteoporosis: a systematic review
}

This article was published in the following Dove Press journal:

Clinical Interventions in Aging

4 January 2017

Number of times this article has been viewed

\author{
Jin-Yu Li ${ }^{1, *}$ \\ Yu-Song Jia ${ }^{1, *}$ \\ Li-Min Chai' \\ Xiao-Hong $\mathrm{Mu}^{\prime}$ \\ Sheng $\mathrm{Ma}^{\mathrm{I}}$ \\ Lin $X u^{\prime}$ \\ $\mathrm{Xu} \mathrm{Wei}{ }^{2}$
}

'Department of Orthopaedics, Dongzhimen Hospital, Beijing University of Chinese Medicine, Beijing, People's Republic of China; ${ }^{2}$ Department of Scientific Research, Wangjing Hospital, China Academy of Chinese Medical Sciences, Beijing, People's Republic of China

*These authors contributed equally to this work
Correspondence: Lin Xu

Department of Orthopaedics,

Dongzhimen Hospital, Beijing University

of Chinese Medicine, No 5 Haiyuncang

Street, Dongcheng District, Beijing

100700, People's Republic of China

Tel +86 I0 840। 557।

Email xulinguke2002@I63.com

Xu Wei

Department of Scientific Research, Wangjing Hospital, China Academy of Chinese Medical Sciences, Huajiadi Street, Chaoyang District, Beijing 100102, People's Republic of China

Tel +86 I3 4887 I6557

Email weixu.007@I63.com
Purpose: The aim is to systematically assess the effectiveness and safety of Chinese herbal formula Erxian decoction (EXD) for treating osteoporosis.

Materials and methods: Six databases were searched from inception through September 17, 2016, without language restriction. All randomized controlled trials of EXD for osteoporosis were included. One or more outcome measures including fracture, change in bone mineral density (BMD), pain symptom improvement, bone biochemical markers, quality of life, adverse event or adverse drug reaction were evaluated. Study selection, data extraction, quality assessment, and data analyses were conducted according to Cochrane standards.

Results: Eight trials including 644 patients investigated the effects of EXD in the treatment of osteoporosis. The methodological quality of the included trials was generally low. The meta-analysis from two trials showed favorable effects of EXD in improving BMD of lumbar spine (mean difference [MD]: 0.05 [0.03, 0.06]; $I^{2}=0 \% ; P<0.00001$ ) and BMD of femoral great trochanter (MD: $0.06[0.02,0.10] ; I^{2}=59 \% ; P=0.005$ ) compared with caltrate tablets. The other meta-analysis from two trials showed beneficial effects of EXD plus caltrate tablets and calcitriol in improving BMD of femoral neck (MD: 0.04 [0.00, 0.09]; $I^{2}=56 \% ; P=0.04$ ), the level of calcium (MD: $0.20[0.15,0.24] ; I^{2}=0 \% ; P<0.00001$ ), and phosphorus (MD: $\left.-0.28[-0.39,-0.17] ; I^{2}=68 \% ; P<0.00001\right)$ compared with caltrate tablets and calcitriol alone. The adverse drug reactions of EXD were mainly slight gastrointestinal symptoms.

Conclusion: The study provides suggestive evidence of the superiority of EXD monotherapy or combination therapy over basic supplements for treating osteoporosis. However, the evidence remains weak. More rigorously designed and measured, randomized double-blind, placebocontrolled trials with larger sample size are needed to verify the current conclusions.

Keywords: Chinese herbal formula, Erxian decoction, osteoporosis, systematic review, metaanalysis

\section{Introduction}

Osteoporosis is a pathological condition that can lead to the reduction of bone mass and the impairment of the bone architecture, which increases the risk of falling and fracture. ${ }^{1,2}$ The disease affects a large amount of the population, especially the elderly and postmenopausal women, being considered a very important problem as a growing concern of aging. ${ }^{3}$ In the last two decades, significant progress has been made in epidemiology studies and in understanding its complex pathogenesis. However, the pathogenesis of osteoporosis is still complex and multifactorial, which makes us put more emphasis on preventing and treating this "silent disease". 4,5 Furthermore, a growing body of research evidence has fostered the development of clinical practice guidelines to monitor and provide appropriate choices for osteoporosis intervention. 
Reducing loss of bone mass, adequately treating clinical symptoms, and preventing osteoporotic fractures are the major therapeutic targets. Therefore, osteoporosis management usually includes pharmacological approaches, physiotherapy interventions, health education measures, and in rare cases, surgical treatment. ${ }^{6}$ Among the treatment strategies, the application of drugs in the management of osteoporosis remains a clinical challenge as a result of patient selection, potential adverse effects or patient adherence. ${ }^{7} \mathrm{Calcium}(\mathrm{Ca})$, vitamin $\mathrm{D}$, bisphosphonates, and calcitonin are the commonly used medications. However, an increasing number of studies demonstrated that using these conventional medicines increased the risk of cardiovascular events. ${ }^{8,9}$ A recent study revealed that $8.5 \%$ reported low adherence to osteoporosis medications and $21.6 \%$ of patients stopped their medication completely over the 2-year follow-up period. ${ }^{10}$ Therefore, there is a need to seek alternative and effective treatment alternatives for osteoporosis. Clinical practice has shown a bright future for Chinese herbal medicine and Chinese herbal formulas in treating osteoporosis. ${ }^{11,12}$

Erxian decoction (EXD), a traditional Chinese herbal formula, has been widely used for treating many chronic diseases, such as osteoporosis, menopausal syndrome, and delayed puberty in the past 60 years. ${ }^{13,14}$ EXD is made up of six herbs, containing Epimedii Folium (Yinyanghuo), Curculiginis Rhizoma (Xianmao), Morindae Officinalis Radix (Bajitian), Anemarrhenae Rhizoma (Zhimu), Phellodendri Chinensis Cortex (Huangbai), and Angelicae Sinensis Radix (Danggui). ${ }^{13}$ Twelve anti-osteoporotic constituents, such as icariin, anemarsaponin BII, and berberine were isolated through bioactivity-guided fractionation. ${ }^{15}$ More and more fundamental researches revealed that EXD had a definite anti-osteoporotic effect similar to estrogen. ${ }^{16-18}$ EXD displayed inhibitory effects on osteoclastic bone resorption, including decreasing the formation of bone resorption pits, the number of multinucleated osteoclasts, and so on..$^{19}$ A recent experimental study further confirmed that EXD could increase bone mass, elevate osteocalcin protein levels in vivo, and promote the self-renewal and osteoblastic differentiation of bone mesenchymal stem cells ex vivo in ovariectomized mice. ${ }^{20}$

A previous systematic review and randomized doubleblind controlled trial has shown that EXD is effective in improving menopausal symptoms. ${ }^{21,22}$ Nevertheless, there is no critically appraised evidence such as systematic reviews or meta-analysis to justify the clinical use of EXD for osteoporosis. Thus, the aim of the current systematic review is to investigate the effectiveness and safety of EXD for the condition.

\section{Materials and methods Search strategy}

Two authors ( $\mathrm{Mu} \mathrm{XH}$ and Ma S) independently performed the literature search using predetermined inclusion/exclusion criteria. A computer-based online search was conducted in the PubMed, Embase, Cochrane Library, Chinese National Knowledge Infrastructure (CNKI), Chinese Scientific Journal Database (VIP), and Wanfang databases. In addition, the reference lists of all obtained articles were examined for additional studies. All the articles were considered without language limitation. All of the searches ended on September 17, 2016. Search terms were Erxian decoction (Er-xian decoction) combined with osteoporosis. The strategy for searching PubMed was listed: ((osteoporosis [MeSH Terms]) AND Erxian decoction) OR Er-xian decoction.

\section{Study selection}

Randomized controlled trials (RCTs) were included, but crossover randomized trials and quasi-randomized trials were excluded. Diagnosis of osteoporosis was established by bone mineral density (BMD) examination. The following interventions were considered for inclusion: EXD versus no treatment or placebo, EXD versus routine anti-osteoporosis medications, EXD plus anti-osteoporosis medications versus anti-osteoporosis medications, EXD plus anti-osteoporosis medications versus placebo plus anti-osteoporosis medications. Modified EXD changes based on traditional Chinese medicine syndrome differentiations were acceptable. The outcome measures included fracture, change in BMD, pain symptom improvement, bone biochemical markers, quality of life, adverse event, or adverse drug reaction (ADR). Any differences in opinion regarding inclusion in the review were resolved by discussion or consulting a third party.

\section{Data extraction and analysis}

Two investigators (Jia YS and Chai LM) independently extracted details of each included study: the principal authors, year of publication, sample sizes, patients' characteristics, details of intervention, details of control interventions, intervention durations, and outcomes for each study. When important data were not available, authors of the relevant study were contacted and requested to provide missing data.

Two authors ( $\mathrm{Li}$ JY and Wei X) assessed the RCTs based on a 7-item quality checklist from the Cochrane Handbook, comprising: 1) random sequence generation; 2) allocation concealment; 3) blinding of participants and personnel; 4) blinding of outcome assessment; 5) incomplete outcome data; 6) selective reporting; and 7) other bias. ${ }^{23}$ The quality 
of RCTs was categorized into three levels: low risk of bias (all the items were assessed as having low risk of bias), high risk of bias (at least one item was assessed as having high risk of bias), and unclear risk of bias (at least one item's risk of bias was in unclear). Discrepancies were resolved by consensus through discussion between the two investigators, if needed, by consulting a third party ( $\mathrm{Xu} \mathrm{L})$.

Data analysis was conducted using the Review Manager 5.2 software provided by the Cochrane Collaboration. The mean difference (MD) was applied for studies using the same scale, and the standardized MD was utilized when the reported scales differed, along with 95\% confidence intervals. For the meta-analysis of the traditional Chinese medicine compound recipe, random-effects model was used to analyze the data. ${ }^{24} P^{2}$ values $\leq 50 \%$ indicated acceptable heterogeneity among studies. The relative strength of treatment efficacy was illustrated by forest plots. When possible, sensitivity analyses were conducted for all outcomes.

\section{Results}

\section{Literature search results}

A flow chart depicted the search process and study selection (Figure 1). A total of 232 articles were initially identified, all of which came from electronic databases. Full text papers of 20 studies were retrieved. Twelve articles were excluded with reasons listed as follows: the intervention included other Chinese herbal formula ( $n=6)$, there was no control group $(n=4)$, and the outcome was not appropriate $(n=2)$. Finally, a total of eight trials including EXD or modified EXD were included..$^{25-32}$ All the papers were published in Chinese. Eight selected trials could be retrieved in the Chinese databases, including CNKI, VIP and Wanfang databases. The characteristics of included trials were listed in Table 1.

\section{Study and participant characteristics}

Eight trials involved a total of 644 patients diagnosed with osteoporosis. The variation in the mean age of participants was 40-70 years. Two trials enrolled middle- and oldaged people, ${ }^{27,30}$ five trials just enrolled postmenopausal women, ${ }^{26,28,29,31,32}$ and one trial did not state the sex distribution of participants. ${ }^{25}$ The different compositions of EXD or modified EXD were presented in Table 2. The controls were routine anti-osteoporosis medications which had six types of groups: 1) caltrate tablets, 2) salmon calcitonin, 3) hormonal treatment, including nilestriol or testosterone propionate, 4) caltrate tablets and calcitriol, 5) caltrate tablets and vitamin D plus alfacalcidol, and 6) elcatonin. The total treatment duration ranged from 2 months to 6 months. The outcome covered BMD, clinical symptoms score, bone metabolism markers, and ADRs.

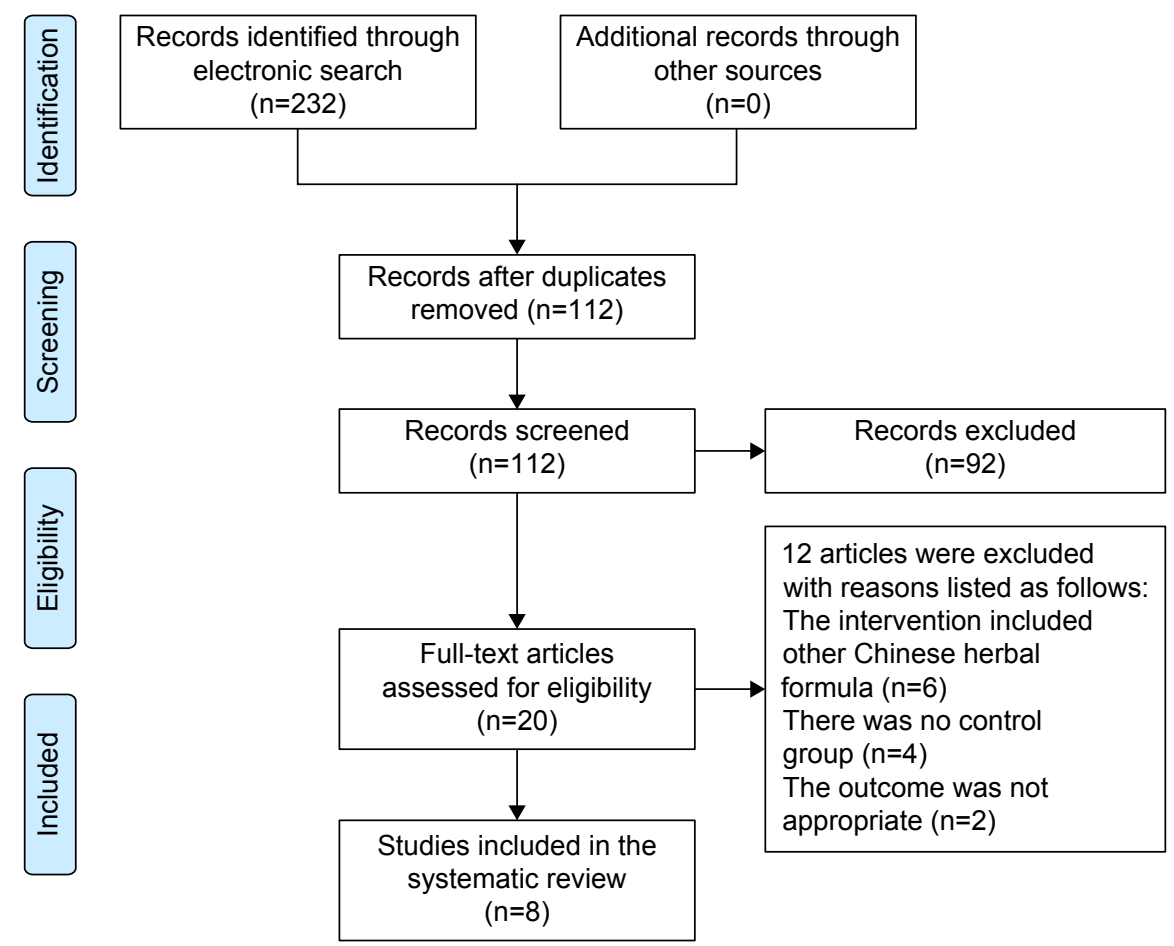

Figure I Study selection process. Databases were PubMed, Embase, Cochrane Library, Chinese National Knowledge Infrastructure (CNKI), Chinese Scientific Journal Database (VIP), and Wanfang databases. 
Table I Characteristics of included trials

\begin{tabular}{|c|c|c|c|c|c|c|c|}
\hline Study ID & $\begin{array}{l}\text { Sample size } \\
\text { (EG/CG) }\end{array}$ & $\begin{array}{l}\text { Age } \\
\text { (years, mean) }\end{array}$ & $\begin{array}{l}\text { Sex } \\
\text { (male/female) }\end{array}$ & $\begin{array}{l}\text { Experimental } \\
\text { group }\end{array}$ & Comparison group & $\begin{array}{l}\text { Duration of } \\
\text { treatment }\end{array}$ & $\begin{array}{l}\text { Outcome } \\
\text { assessment }\end{array}$ \\
\hline Wu et $\mathrm{al}^{25}$ & $58(30 / 28)$ & $\begin{array}{l}\text { EG: } 68.65 \pm 7.07 \\
\text { CG: } 67.91 \pm 7.37\end{array}$ & NR & $\begin{array}{l}\text { EXD (a dose/d, Qd, } \\
\text { oral administration) }\end{array}$ & $\begin{array}{l}\text { Caltrate tablets }(600 \mathrm{mg}, \mathrm{Qd} \text {, } \\
\text { oral administration) }\end{array}$ & 6 months & $\begin{array}{l}\text { BMD (LS, FGT) } \\
\mathrm{Ca}, \mathrm{P}, \mathrm{ALP}\end{array}$ \\
\hline $\begin{array}{l}\text { Zhu and } \\
\mathrm{Gu}^{26}\end{array}$ & $70(35 / 35)$ & $\begin{array}{l}\text { EG: } 61.2 \pm 5.6 \\
\text { CG: } 60.4 \pm 5.8\end{array}$ & $\begin{array}{l}\text { Postmenopausal } \\
\text { women }\end{array}$ & $\begin{array}{l}\text { EXD (a dose/d, } \\
\text { Bid, oral } \\
\text { administration) + CG }\end{array}$ & $\begin{array}{l}\text { Salmon Calcitonin (50 IU } \\
\text { every day, intramuscular } \\
\text { injection) }\end{array}$ & 3 months & $\begin{array}{l}\text { BMD (LS) } \\
\text { ALP, BGP, } \\
\text { CT, } E_{2}\end{array}$ \\
\hline $\mathrm{Gao}^{27}$ & $78(39 / 39)$ & $\begin{array}{l}\text { EG: } 42.3 \pm 5.6 \\
\text { CG: } 40.7 \pm 4.9\end{array}$ & $\begin{array}{l}E G: 19 / 20 \\
C G: 21 / 18\end{array}$ & $\begin{array}{l}\text { EXD (a dose/d, oral } \\
\text { administration) }\end{array}$ & $\begin{array}{l}\text { Women: Nilestriol ( } 2 \sim 4 \mathrm{mg} \\
\text { every } 2 \text { weeks, oral } \\
\text { administration) } \\
\text { Men: testosterone propionate } \\
\text { (20 } 25 \mathrm{mg} \text { every } 2 \text { weeks, } \\
\text { intramuscular) }\end{array}$ & 2 months & $\begin{array}{l}\text { BMD (LS, FN, } \\
\text { WA, FGT) }\end{array}$ \\
\hline $\operatorname{Liu}^{28}$ & $138(69 / 69)$ & $56.2 \pm 9.3$ & $\begin{array}{l}\text { Postmenopausal } \\
\text { women }\end{array}$ & $\begin{array}{l}\text { Modified EXD } \\
\text { (a dose/d, oral } \\
\text { administration) + CG }\end{array}$ & $\begin{array}{l}\text { Caltrate tablets }(600 \mathrm{mg} \text {, } \\
\text { Qd, oral administration) and } \\
\text { calcitriol }(0.25 \mu \mathrm{g} \text {, Qd, oral } \\
\text { administration) }\end{array}$ & 3 months & $\begin{array}{l}\text { BMD (LS, FN) } \\
\mathrm{Ca}, \mathrm{P}, \mathrm{ALP} \\
\text { ADR }\end{array}$ \\
\hline Jiang et $\mathrm{a}^{29}$ & $70(35 / 35)$ & $\begin{array}{l}E G: 64.64 \pm 8.73 \\
C G: 69.4 I \pm 5.21\end{array}$ & $\begin{array}{l}\text { Postmenopausal } \\
\text { women }\end{array}$ & $\begin{array}{l}\text { Modified EXD } \\
\text { (a dose/d, oral } \\
\text { administration) + CG }\end{array}$ & $\begin{array}{l}\text { Caltrate tablets }(600 \mathrm{mg} \text {, } \\
\text { Qd, oral administration) and } \\
\text { calcitriol }(0.25 \mu \mathrm{g} \text {, Bid, oral } \\
\text { administration) }\end{array}$ & 3 months & $\begin{array}{l}\text { BMD (LS, FN) } \\
\text { Ca, P, ALP, } \\
\text { ADR }\end{array}$ \\
\hline $\operatorname{Liu}^{30}$ & $70(35 / 35)$ & $\begin{array}{l}\text { EG: } 42.5 \pm 5.1 \\
\text { CG: } 43.6 \pm 5.4\end{array}$ & $\begin{array}{l}E G: 15 / 20 \\
C G: 16 / 19\end{array}$ & $\begin{array}{l}\text { Modified EXD } \\
\text { (a dose/d, Bid, oral } \\
\text { administration) }\end{array}$ & $\begin{array}{l}\text { Caltrate tablets ( } 600 \mathrm{mg}, \mathrm{Qd} \text {, } \\
\text { oral administration) }\end{array}$ & 2 months & $\begin{array}{l}\text { BMD (LS, FN, } \\
\text { WA, FGT) } \\
\text { VAS score } \\
\text { Ca, P, ALP }\end{array}$ \\
\hline Xu et $\mathrm{al}^{31}$ & $80(40 / 40)$ & $\begin{array}{l}\text { EG: } 63.19 \pm 5.13 \\
\text { CG: } 64.32 \pm 6.34\end{array}$ & $\begin{array}{l}\text { Postmenopausal } \\
\text { women }\end{array}$ & $\begin{array}{l}\text { Modified EXD } \\
\text { (a dose/d, oral } \\
\text { administration) + CG }\end{array}$ & $\begin{array}{l}\text { Caltrate tablets ( } 600 \mathrm{mg} \text {, } \\
\text { Qd, oral administration), } \\
\text { Vitamin D (I } 25 \mathrm{IU}, \mathrm{Qd}, \\
\text { oral administration) and } \\
\text { Alfacalcidol ( } 0.25 \mu \mathrm{g} \text {, Qd, oral } \\
\text { administration) }\end{array}$ & 3 months & $\begin{array}{l}\text { BMD (FN) } \\
\text { ALP, } E_{2}\end{array}$ \\
\hline Ye et $\mathrm{al}^{32}$ & $80(40 / 40)$ & $\begin{array}{l}E G: 48.40 \pm 7.58 \\
\text { CG: } 47.10 \pm 3.11\end{array}$ & $\begin{array}{l}\text { Postmenopausal } \\
\text { women }\end{array}$ & $\begin{array}{l}\text { EXD (a dose/d, } \\
\text { Bid, oral } \\
\text { administration) + CG }\end{array}$ & $\begin{array}{l}\text { Elcatonin (I0 IU per time, } \\
\text { twice a week, intramuscular } \\
\text { injection) }\end{array}$ & 3 months & $\begin{array}{l}\text { BMD (LS, tibia) } \\
\text { VAS score, } \\
\text { ADR }\end{array}$ \\
\hline
\end{tabular}

Abbreviations: EG, experimental group; CG, comparison group; NR, not reported; EXD, Erxian decoction; LS, lumbar spine; FN, femoral neck; WA, Ward's area; FGT, femoral great trochanter; BMD, bone mineral density; Ca, calcium; P, phosphorus; CT, calcitonin; $E_{2}$, estradiol; ADR, adverse drug reaction; VAS, visual analog scale; ALP, alkaline phosphatase; BGP, bone gla protein.

\section{Methodological quality of included trials}

The methodological quality assessments were summarized in Table 3. All of the included trials were evaluated and deemed to be of generally poor methodological quality. Two trials stated that the method of random number table was used, ${ }^{26,29}$ one trial described the random allocation using SPSS software. ${ }^{32}$ The others only mentioned that "subjects were randomized into two groups", but did not report the detailed methods for sequence generation. None of the trials reported allocation concealment and used matched placebo to blind participants and practitioners. The number of dropout or withdrawal cases and intention-to-treat analysis were not mentioned, so the item "incomplete outcome data" was not identified. Selective reporting was unclear because the protocols of all the trials were not accessible. The baseline information on the age, sex, and the outcomes was not adequate in the four trials..$^{25,28,29,31}$

\section{Fracture prevention and quality of life}

None of the trials reported the fracture incidence and quality of life as the observed outcomes.

\section{BMD}

The trials that tested the BMD values in different parts of the body were analyzed.

1) Improvement of BMD in lumbar spine. Seven trials studied the benefits of improved BMD in lumbar spine and two meta-analyses could be performed. ${ }^{25-30,32}$ Two studies including 128 participants compared the effect of EXD with caltrate tablets to measure the outcome. ${ }^{25,30} \mathrm{~A}$ meta-analysis showed a significant beneficial effect of EXD compared with caltrate tablets in improving BMD of lumbar spine (MD: 0.05 $[0.03,0.06] ; I^{2}=0 \% ; P<0.00001$ ) (Figure 2). In addition, two studies including 208 participants compared the effect of EXD plus caltrate tablets and calcitriol with caltrate tablets 
Table 2 Composition of formula

\begin{tabular}{|c|c|c|}
\hline Study ID & Formula & Composition of formula \\
\hline Wu et $\mathrm{al}^{25}$ & EXD & NR \\
\hline $\begin{array}{l}\text { Zhu and } \\
\mathrm{Gu}^{26}\end{array}$ & EXD & $\begin{array}{l}\text { Epimedii Folium (Yinyanghuo) } 20 \mathrm{~g} \text {, Curculiginis Rhizoma (Xianmao) } 20 \mathrm{~g} \text {, Morindae Officinalis Radix } \\
\text { (Bajitian) } 15 \mathrm{~g} \text {, Anemarrhenae Rhizoma (Zhimu) } 12 \mathrm{~g} \text {, Phellodendri Chinensis Cortex (Huangbai) } 15 \mathrm{~g} \text {, } \\
\text { Angelicae Sinensis Radix (Danggui) } 10 \mathrm{~g}\end{array}$ \\
\hline $\mathrm{Gao}^{27}$ & EXD & $\begin{array}{l}\text { Epimedii Folium (Yinyanghuo) } 30 \mathrm{~g} \text {, Curculiginis Rhizoma (Xianmao) } 30 \mathrm{~g} \text {, Morindae Officinalis Radix } \\
\text { (Bajitian) } 15 \mathrm{~g} \text {, Anemarrhenae Rhizoma (Zhimu) } 10 \mathrm{~g} \text {, Phellodendri Chinensis Cortex (Huangbai) } 10 \mathrm{~g} \text {, } \\
\text { Angelicae Sinensis Radix (Danggui) } 10 \mathrm{~g}\end{array}$ \\
\hline $\operatorname{Liu}^{28}$ & Modified EXD & $\begin{array}{l}\text { Epimedii Folium (Yinyanghuo) } 15 \mathrm{~g} \text {, Curculiginis Rhizoma (Xianmao) } 15 \mathrm{~g} \text {, Morindae Officinalis } \\
\text { Radix (Bajitian) } 15 \mathrm{~g} \text {, Anemarrhenae Rhizoma (Zhimu) } 10 \mathrm{~g} \text {, Angelicae Sinensis Radix (Danggui) } 10 \mathrm{~g} \text {, } \\
\text { Testudinis Carapacis Et Plastri Colla (Guijiajiao) } 12 \mathrm{~g} \text {, Corni Fructus (Shanzhuyu) } 12 \mathrm{~g} \text {, Lycii Fructus } \\
\text { (Gouqi) } 12 \mathrm{~g} \text {, Salviae Miltiorrhizae Radix Et Rhizoma (Danshen) } 15 \mathrm{~g}\end{array}$ \\
\hline Jiang et $\mathrm{al}^{29}$ & Modified EXD & $\begin{array}{l}\text { Epimedii Folium (Yinyanghuo) } 15 \mathrm{~g} \text {, Curculiginis Rhizoma (Xianmao) } 15 \mathrm{~g} \text {, Morindae Officinalis Radix } \\
\text { (Bajitian) } 10 \mathrm{~g} \text {, Anemarrhenae Rhizoma (Zhimu) } 15 \mathrm{~g} \text {, Phellodendri Chinensis Cortex (Huangbai) } 10 \mathrm{~g} \text {, } \\
\text { Angelicae Sinensis Radix (Danggui) } 15 \mathrm{~g} \text {, Pyritum (Zirantong) } 10 \mathrm{~g}\end{array}$ \\
\hline $\operatorname{Liu}^{30}$ & Modified EXD & $\begin{array}{l}\text { Epimedii Folium (Yinyanghuo) } 15 \mathrm{~g} \text {, Curculiginis Rhizoma (Xianmao) } 10 \mathrm{~g} \text {, Morindae Officinalis Radix } \\
\text { (Bajitian) } 15 \mathrm{~g} \text {, Anemarrhenae Rhizoma (Zhimu) } 15 \mathrm{~g} \text {, Phellodendri Chinensis Cortex (Huangbai) } 10 \mathrm{~g} \text {, } \\
\text { Angelicae Sinensis Radix (Danggui) } 5 \mathrm{~g} \text {, Corni Fructus (Shanzhuyu) } 10 \mathrm{~g} \text {, Rehmanniae Radix Praeparata } \\
\text { (Shudihuang) } 30 \mathrm{~g} \text {, Dioscoreae Rhizoma (Shanyao) } 15 \mathrm{~g} \text {, Drynariae Rhizoma (Gusuibu) } 15 \mathrm{~g} \text {, Cyathulae } \\
\text { Radix (Chuanniuxi) } 10 \mathrm{~g} \text {, Glycyrrhizae Radix Et Rhizoma (Gancao) } 3 \mathrm{~g} \text {, Jujubae Fructus (Dazao) } 30 \mathrm{~g}\end{array}$ \\
\hline Xu et $\mathrm{al}^{|3|}$ & Modified EXD & $\begin{array}{l}\text { Epimedii Folium (Yinyanghuo) } 20 \mathrm{~g} \text {, Curculiginis Rhizoma (Xianmao) } 20 \mathrm{~g} \text {, Morindae Officinalis Radix } \\
\text { (Bajitian) } 20 \mathrm{~g} \text {, Anemarrhenae Rhizoma (Zhimu) } 15 \mathrm{~g} \text {, Phellodendri Chinensis Cortex (Huangbai) } \\
10 \mathrm{~g} \text {, Angelicae Sinensis Radix (Danggui) } 15 \mathrm{~g} \text {, Testudinis Carapacis Et Plastri Colla (Guijiajiao) } 10 \mathrm{~g} \text {, } \\
\text { Corni Fructus (Shanzhuyu) } 10 \mathrm{~g} \text {, Lycii Fructus (Gouqi) } 10 \mathrm{~g} \text {, Salviae Miltiorrhizae Radix Et Rhizoma } \\
\text { (Danshen) } 10 \mathrm{~g}\end{array}$ \\
\hline $\begin{array}{l}\text { Zhu and } \\
\mathrm{Gu}^{32}\end{array}$ & EXD & $\begin{array}{l}\text { Epimedii Folium (Yinyanghuo) } 15 \mathrm{~g} \text {, Curculiginis Rhizoma (Xianmao) } 15 \mathrm{~g} \text {, Morindae Officinalis Radix } \\
\text { (Bajitian) } 10 \mathrm{~g} \text {, Anemarrhenae Rhizoma (Zhimu) } 6 \mathrm{~g} \text {, Phellodendri Chinensis Cortex (Huangbai) } 6 \mathrm{~g} \text {, } \\
\text { Angelicae Sinensis Radix (Danggui) } 10 \mathrm{~g}\end{array}$ \\
\hline
\end{tabular}

Abbreviations: EXD, Erxian decoction; NR, not reported.

and calcitriol. ${ }^{28,29}$ A pooled analysis indicated that there was no significant difference between the integrative therapy and routine treatment drugs in improving BMD of lumbar spine (MD: $0.08[-0.03,0.18] ; P=91 \% ; P=0.15$ ) (Figure 3).

The other three trials were not included in metaanalyses. ${ }^{26,27,32}$ One study by Zhu and $\mathrm{Gu}$ indicated EXD plus salmon calcitonin was better than the use of salmon calcitonin alone over a period of three months $(P<0.05){ }^{26}$ Anther study found a greater improvement in the EXD group compared to hormonal treatment over a period of two months
$(P<0.05) .{ }^{27}$ Another study demonstrated that the result of the EXD plus elcatonin group was significantly better than elcatonin monotherapy in enhancing the BMD in lumbar spine L2 to L4 after three months $(P<0.001){ }^{32}$

2) Improvement of BMD in femoral neck. Five trials studied BMD in femoral neck, and one meta-analysis was conducted to evaluate the effect of the Chinese herbal formula. ${ }^{27-31}$ The meta-analysis from two trials demonstrated that EXD plus caltrate tablets and calcitriol was superior to caltrate tablets and calcitriol alone in improving

Table 3 Methodological quality of the included trials

\begin{tabular}{|c|c|c|c|c|c|c|c|}
\hline Study ID & $\begin{array}{l}\text { Random sequence } \\
\text { generation }\end{array}$ & $\begin{array}{l}\text { Allocation } \\
\text { concealment }\end{array}$ & $\begin{array}{l}\text { Blinding of participants } \\
\text { and personnel }\end{array}$ & $\begin{array}{l}\text { Blinding of outcome } \\
\text { assessment }\end{array}$ & $\begin{array}{l}\text { Incomplete } \\
\text { outcome data }\end{array}$ & $\begin{array}{l}\text { Selective } \\
\text { reporting }\end{array}$ & $\begin{array}{l}\text { Other } \\
\text { bias }\end{array}$ \\
\hline Wu et $\mathrm{al}^{25}$ & - & ? & - & $?$ & $?$ & $?$ & ? \\
\hline Zhu and $\mathrm{Gu}^{26}$ & + & ? & - & $?$ & $?$ & $?$ & + \\
\hline $\mathrm{Gao}^{27}$ & - & $?$ & - & $?$ & $?$ & $?$ & + \\
\hline $\operatorname{Liu}^{28}$ & - & ? & - & ? & $?$ & $?$ & $?$ \\
\hline Jiang et $\mathrm{al}^{29}$ & + & ? & - & $?$ & $?$ & $?$ & ? \\
\hline $\operatorname{Liu}^{30}$ & - & ? & - & $?$ & $?$ & $?$ & + \\
\hline$X u$ et $\mathrm{al}^{3 \mathrm{I}}$ & - & ? & - & ? & $?$ & $?$ & ? \\
\hline Ye et $\mathrm{al}^{32}$ & + & ? & - & ? & $?$ & $?$ & + \\
\hline
\end{tabular}

Notes: “+”, low risk of bias; “-”, high risk of bias; “?”, unclear risk of bias. 


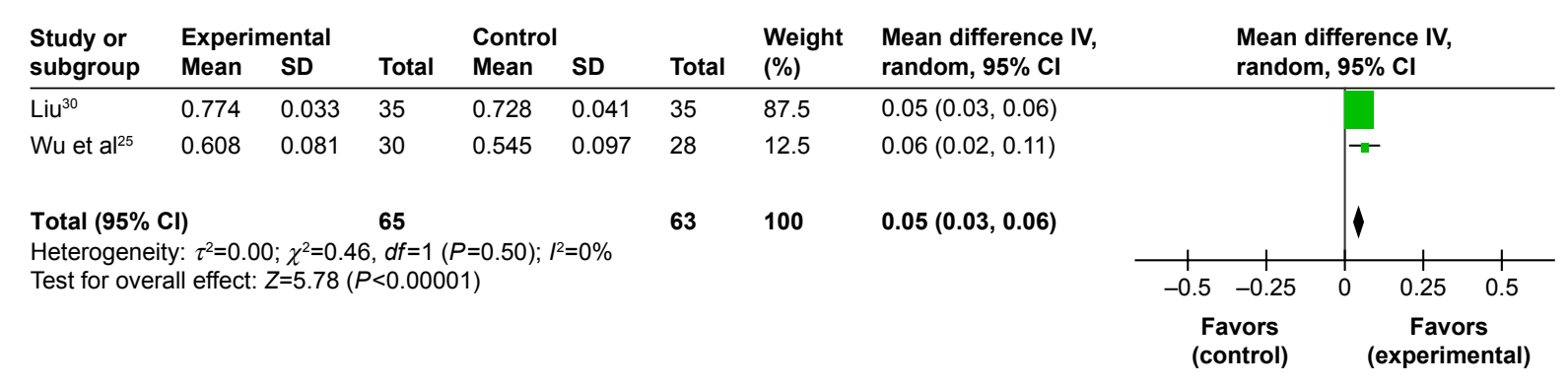

Figure 2 Forest plot of comparison between EXD and caltrate tablets. The outcome was BMD in lumbar spine.

Abbreviations: EXD, Erxian decoction; BMD, bone mineral density; $\mathrm{Cl}$, confidence interval; SD, standard deviation; $\mathrm{df}$, degrees of freedom.

BMD of femoral neck (MD: $0.04[0.00,0.09] ; I^{2}=56 \%$; $P=0.04$ ) (Figure 4). ${ }^{28,29}$

The other three trials were not included in metaanalyses. ${ }^{27,30,31}$ Gao's study showed EXD alone had a significant advantage compared to hormonal treatment in enhancing BMD of femoral neck $(P<0.05) .{ }^{27}$ Another study showed a significant treatment effect of modified EXD compared with caltrate tablets $(P=0.009) \cdot{ }^{30} \mathrm{Xu}$ et al confirmed the effect of combination therapy (modified EXD plus anti-osteoporosis medicine) was better than anti-osteoporosis medicine alone after three months of therapy $(P<0.05){ }^{31}$

3) Improvement of BMD in femoral great trochanter. Three trials observed the effect of BMD in femoral great trochanter. ${ }^{25,27,30}$ One meta-analysis could be performed. ${ }^{25,30}$ The meta-analysis showed there is significant beneficial effect in EXD group compared to caltrate tablets group (MD: 0.06 [0.02, 0.10 ]; $P=59 \% ; P=0.005$ ) (Figure 5). The remaining trial reported that the EXD group experienced more benefits than the hormonal treatment group $(P<0.05){ }^{27}$

4) Improvement of BMD in Ward's area. Two trials observed the effect of BMD in Ward's area. ${ }^{27,30}$ One trial reported that EXD group experienced more benefits than the hormonal treatment group after two months $(P<0.05){ }^{27}$ The other trial also found modified EXD showed more improvement than caltrate tablets after two months of treatment $(P<0.001){ }^{30}$

5) Improvement of BMD in tibia. Only one trial assessed the effect of BMD in tibia. ${ }^{32}$ The result revealed that the curative effect experienced by the EXD plus elcatonin group was better than the elcatonin group after three months $(P<0.001)$.

\section{Pain symptom}

Two trials used the pain score as the outcome. ${ }^{30,32}$ One trial showed that the modified EXD was better than caltrate tablets in reducing pain (visual analog scale score) after two months $(P<0.001)$. The other trial evaluated the pain-relieving effect of EXD plus elcatonin. ${ }^{32}$ After three-month treatment, EXD plus elcatonin could significantly improve the pain symptom of osteoporosis compared with elcatonin alone $(P<0.001)$.

\section{Bone biochemical markers}

The trials that measured the blood $\mathrm{Ca}$, phosphorus $(\mathrm{P})$, alkaline phosphatase (ALP), bone gla protein (BGP), calcitonin $(\mathrm{CT})$, and estradiol $\left(\mathrm{E}_{2}\right)$ were analyzed.

1) Improvement of Ca, P, ALP. Five trials analyzed the level of blood $\mathrm{Ca}$ and two meta-analyses were conducted. . $^{25,26,28,30}$ No statistical difference in blood Ca (MD: $-0.13[-0.28$, $0.01] ; I^{2}=38 \% ; P=0.06$ ), blood P (MD: 0.13 [-0.19, 0.49]; $\left.I^{2}=91 \% ; P=0.39\right)$, and blood ALP (MD: $-1.21[-5.54,3.12]$; $I^{2}=0 \% ; P=0.59$ ) were found between the EXD group and caltrate tablets group. However, EXD plus caltrate tablets and calcitriol significantly increased the level of blood $\mathrm{Ca}$ (MD: $\left.0.20[0.15,0.24] ; I^{2}=0 \% ; P<0.00001\right)$ and lowered the level of blood P (MD: $-0.28[-0.39,-0.17] ; P=68 \%$; $P<0.00001)$ compared with caltrate tablets and calcitriol

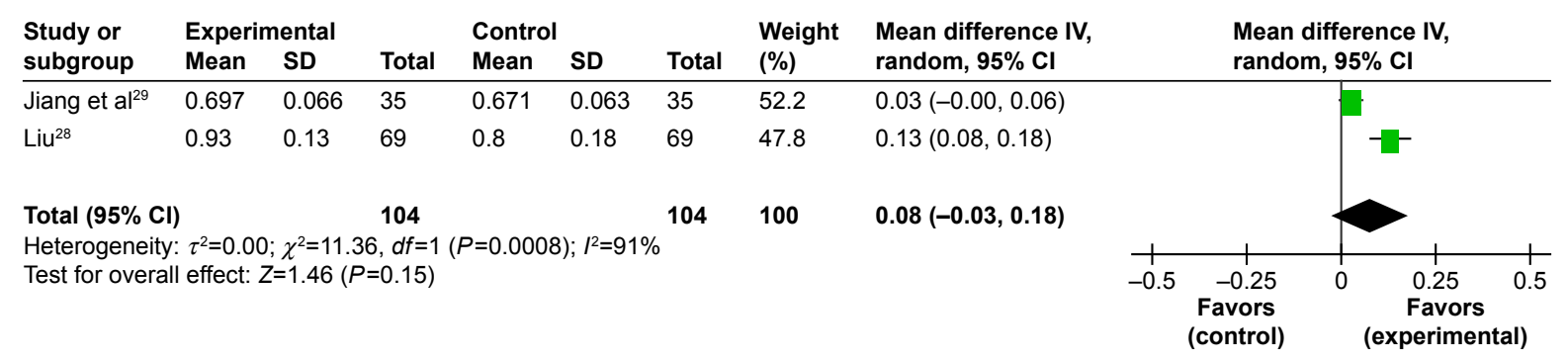

Figure 3 Forest plot of comparison between EXD plus caltrate tablets and calcitriol versus caltrate tablets and calcitriol. The outcome was BMD in lumbar spine. Abbreviations: EXD, Erxian decoction; BMD, bone mineral density; $\mathrm{Cl}$, confidence interval; SD, standard deviation; df, degrees of freedom. 


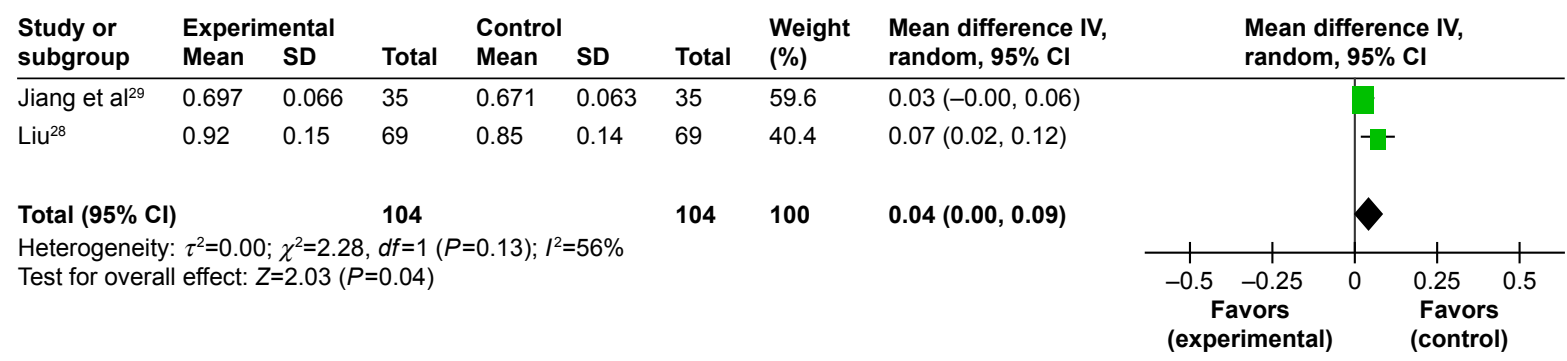

Figure 4 Forest plot of comparison between EXD plus caltrate tablets and calcitriol versus caltrate tablets and calcitriol. The outcome was BMD in femoral neck. Abbreviations: EXD, Erxian decoction; BMD, bone mineral density; $\mathrm{Cl}$, confidence interval; SD, standard deviation; $\mathrm{df}$, degrees of freedom.

based on the meta-analysis. No statistical differences in blood ALP (MD: $-1.87[-7.58,3.84] ; P=0 \% ; P=0.52$ ) were found between the integrative therapy group and routine treatment drugs group.

The other two trials were not analyzed in meta-analyses. ${ }^{26,31}$ One trial showed a statistically significant difference in favor of EXD plus salmon calcitonin in improving the level of ALP compared to salmon calcitonin $(P<0.05)$ after three months. ${ }^{26}$ The other showed a statistically significant difference in favor of the combination therapy in improving the level of ALP compared to caltrate tablets and vitamin D plus alfacalcidol $(P<0.05)$ after three months. ${ }^{31}$

2) Improvement of $B G P, C T, E_{2}$. Two trials analyzed the level of blood BGP, CT, $\mathrm{E}_{2}$ and no meta-analysis could be conducted. ${ }^{26,31}$ The statistical data indicated that EXD plus salmon calcitonin was better than salmon calcitonin alone with regard to increasing the level of BGP $(P<0.05)$, CT $(P<0.05), \mathrm{E}_{2}(P<0.01)$ after three months ${ }^{26}$ The other data also indicated that the combination therapy was better than caltrate tablets and vitamin $D$ plus alfacalcidol with regard to increasing the level of $\mathrm{E}_{2}(P<0.05)$ after three months. ${ }^{31}$

\section{Adverse effects}

Three trials (3/8) reported the ADRs..$^{28,29,32}$ One trial demonstrated 1 case with abdominal distension $(1 / 69,1.45 \%)$, 3 cases $(2 / 69,4.35 \%)$ with constipation and 2 cases $(2 / 69$, $2.90 \%$ ) with stomach upset in the modified EXD plus caltrate tablets and calcitriol group. ${ }^{28}$ However, the occurrence of
ADRs was higher in the caltrate tablets and calcitriol group $(P<0.05)$, including 5 cases with abdominal distension $(5 / 69,7.25 \%), 7$ cases $(7 / 69,10.14 \%)$ with constipation, and 6 cases $(6 / 69,8.70 \%)$ with stomach upset. Another trial exhibited 1 case with diarrhea $(1 / 40,2.5 \%)$ in the EXD group, 1 case with facial flushing $(1 / 40,2.5 \%)$, and 1 case with nausea $(1 / 40,2.5 \%)$ in the elcatonin group. ${ }^{32}$ And the adverse effects were not serious in the Chinese medicine group. No ADRs were found in another trial. ${ }^{29}$ The remaining five trials did not mention whether the adverse events were monitored.

\section{Publication bias}

The funnel plots were not conducted due to the inadequate number of included osteoporosis studies.

\section{Discussion}

Plant-based therapies including traditional Chinese medicines have long been used to prevent and treat osteoporosis and have received extensive attention. ${ }^{33-35}$ According to the theory of Chinese medicine, Shen-Jing deficiency (Kidney essence) plays an important part in the development of osteoporosis and is better for diagnosing osteoporosis. ${ }^{36}$ Therefore, kidney-tonifying herbs or herbal formula contribute to treating both the disease and the Chinese medicine syndromes. ${ }^{36,37}$ Among the several Chinese medicine prescriptions, EXD is one of the most representative formulas reflecting kidneytonifying principle. ${ }^{38}$ To our knowledge, this is the first

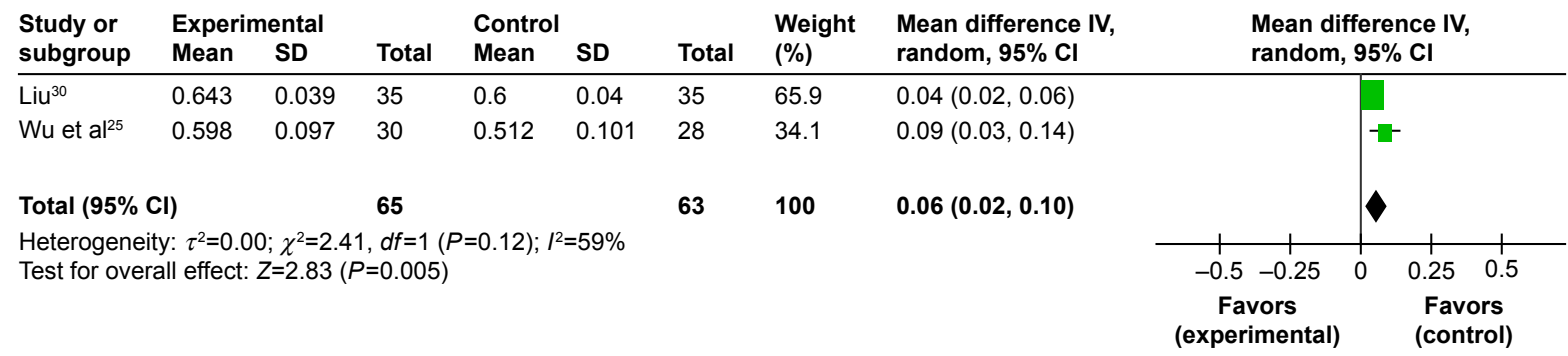

Figure 5 Forest plot of comparison between EXD and caltrate tablets. The outcome was BMD in femoral great trochanter.

Abbreviations: EXD, Erxian decoction; BMD, bone mineral density; $\mathrm{Cl}$, confidence interval; SD, standard deviation; $d f$, degrees of freedom. 
systematic review on EXD for osteoporosis with regard to different outcomes.

A detailed analysis was performed based on the different interventions, controls, and outcomes. One aggregated result indicated that EXD was more effective for participants in increasing BMD of lumbar spine and femoral great trochanter than for those in the caltrate tablets group in only two studies. The other aggregated result showed that EXD plus caltrate tablets and calcitriol was more effective in increasing BMD of femoral neck and improving the level of blood $\mathrm{Ca}$ and $\mathrm{P}$ than caltrate tablets and calcitriol in only two studies. Additionally, two independent trials showed that modified EXD or EXD plus elcatonin significantly improved pain symptoms in patients with osteoporosis. The ADR of EXD might be abdominal distension, constipation, diarrhea, and stomach upset.

In our review, quality of life was not observed in the previous studies. Also, there was insufficient evidence to support or refute the value of EXD monotherapy or combination therapy compared with other active therapies alone because of the limited number of studies. A randomized double-blind, placebo-controlled trial was not found in the literature. Thus, the definite effect of Chinese medicine still needs to be proven by scientific research. Placebo control is of equal importance as active control in clinical trials of Chinese medicine. ${ }^{39}$ Further studies should first compare EXD with placebo or no treatment. More standard therapies, such as bisphosphonate or exercise, need to be considered as a control. Our results indicated that a minimal valid duration of EXD for osteoporosis might be at least 2 months. Nevertheless, the longer duration may achieve better gains. Unlike diabetes and hypertension, conventional drugs for osteoporosis can bring persistent structural benefits. ${ }^{40,41}$ But so far the longterm effects and safety of EXD are still unknown.

Before accepting the above findings, the following limitations should be noted. First, in all the articles published in People's Republic of China, there were no data about the effect of EXD for treating osteoporosis in other countries. Therefore, the potential selection bias could not be ruled out. Second, the majority of studies failed to report the random method, did not use blinding, did not describe the number and the reason of withdrawal, and did not register the study protocol. Similar to the several systematic reviews of alternative medicine for osteoporosis, the methodological quality of RCTs is the major problem affecting the efficacy evaluation. ${ }^{42,43}$ The researchers of Chinese medicine should attach more importance to improve reporting quality using the Consolidated Standards of Reporting Trials (CONSORT) statement. ${ }^{44}$ Third, our results may be affected by the modified herbs, dosing parameters of EXD, and treatment duration. Fourth, the information on the adverse events or ADR is inadequate. The safety of EXD needs to be rigorously monitored in future clinical trials. Due to the poor quality of the previous studies, the results of our review are likely to be influenced by many factors.

\section{Conclusion}

The present meta-analysis proved that EXD monotherapy or EXD plus caltrate tablets and calcitriol for osteoporosis may have beneficial effects on the improvement of BMD at anatomical sites. EXD plus caltrate tablets and calcitriol can significantly improve the level of $\mathrm{Ca}$ and $\mathrm{P}$. As a result of the methodological drawbacks in the previous studies, more rigorously designed and measured, randomized double-blind, placebo-controlled trials with larger sample size are needed to verify the current conclusions.

\section{Acknowledgments}

The authors are thankful for the financial support from National Natural Science Foundation of China (81503601) and Science and Technology Program of Beijing Administration of Traditional Chinese Medicine (JJ2015-57). The authors would like to thank Yukun Yin from Indiana University Purdue University Indianapolis for revising the English language of this paper.

\section{Disclosure}

The authors report no conflicts of interest in this work.

\section{References}

1. Hernlund E, Svedbom A, Ivergård M, et al. Osteoporosis in the European Union: medical management, epidemiology and economic burden. A report prepared in collaboration with the International Osteoporosis Foundation (IOF) and the European Federation of Pharmaceutical Industry Associations (EFPIA). Arch Osteoporos. 2013;8:136.

2. Lin X, Xiong D, Peng YQ, et al. Epidemiology and management of osteoporosis in the People's Republic of China: current perspectives. Clin Interv Aging. 2015;10:1017-1033.

3. Abobului M, Berghea F, Vlad V, et al. Evaluation of adherence to antiosteoporosis treatment from the socio-economic context. J Med Life. 2015;8 Spec Issue:119-123.

4. Rosen CJ. The Epidemiology and Pathogenesis of Osteoporosis. Endotext. 2014.

5. Mafi Golchin M, Heidari L, Ghaderian SM, Akhavan-Niaki H. Osteoporosis: a silent disease with complex genetic contribution. $J$ Genet Genomics. 2016;43(2):49-61.

6. Paolucci T, Saraceni VM, Piccinini G. Management of chronic pain in osteoporosis: challenges and solutions. J Pain Res. 2016;9: 177-186.

7. Bolland MJ, Grey A, Avenell A, Gamble GD, Reid IR. Calcium supplements with or without vitamin D and risk of cardiovascular events: reanalysis of the Women's Health Initiative limited access dataset and meta-analysis. BMJ. 2011;342:d2040.

8. Maraka S, Kennel KA. Bisphosphonates for the prevention and treatment of osteoporosis. BMJ. 2015;351:h3783. 
9. Wang JC, Chien WC, Chung CH, Liao WI, Tsai SH. Adverse cardiovascular effects of nitrogen-containing bisphosphonates in patients with osteoporosis: a nationwide population-based retrospective study. Int $J$ Cardiol. 2016;215:232-237.

10. Clark EM, Gould VC, Tobias JH, Home R. Natural history, reasons for, and impact of low/non-adherence to medications for osteoporosis in a cohort of community-dwelling older women already established on medication: a 2-year follow-up study. Osteoporos Int. 2016;27(2):579-590.

11. Wang L, Li Y, Guo Y, et al. Herba Epimedii: an ancient Chinese herbal medicine in the prevention and treatment of osteoporosis. Curr Pharm Des. 2016;22(3):328-349.

12. Siu WS, Wong HL, Lau CP, et al. The effects of an antiosteoporosis herbal formula containing epimedii herba, ligustri lucidi fructus and psoraleae fructus on density and structure of rat long bones under tail-suspension, and its mechanisms of action. Phytother Res. 2013;27(4):484-492.

13. Li JJ, Li JT, Fu JP. Erxian tang-introduction of a Chinese herbal formula, clinical practice, and experimental studies. Chin J Integr Med. 2007; 13(1):67-73.

14. Zhu Z, Li L, Jin X, Fang J, Zhang D. Er-Xian decoction, a traditional Chinese herbal formula, intervening early in hypothalamic-pituitary axis of male rats with delayed puberty. Pharmacogn Mag. 2014;10(40): 517-521.

15. Qin L, Han T, Zhang Q, et al. Antiosteoporotic chemical constituents from Er-Xian decoction, a traditional Chinese herbal formula. $J$ Ethnopharmacol. 2008;118(2):271-279.

16. Nian H, Qin LP, Zhang QY, Zheng HC, Yu Y, Huang BK. Antiosteoporotic activity of Er-Xian decoction, a traditional Chinese herbal formula, in ovariectomized rats. J Ethnopharmacol. 2006;108(1):96-102.

17. Xue L, Wang Y, Jiang Y, et al. Comparative effects of Er-Xian decoction, epimedium herbs, and icariin with estrogen on bone and reproductive tissue in ovariectomized rats. Evid Based Complement Alternat Med. 2012;2012:241416.

18. Wong KC, Lee KS, Luk HK, et al. Er-xian Decoction exerts estrogenlike osteoprotective effects in vivo and in vitro. Am J Chin Med.2014; 42(2):409-426.

19. Xue L, Jiao L, Wang Y, et al. Effects and interaction of icariin, curculigoside, and berberine in er-xian decoction, a traditional Chinese medicinal formula, on osteoclastic bone resorption. Evid Based Complement Alternat Med. 2012;2012:490843.

20. Liu S, Huang J, Wang J, et al. Er-Xian Decoction stimulates osteoblastic differentiation of bone mesenchymal stem cells in ovariectomized mice and its gene profile analysis. Stem Cells Int. 2016;2016: 4079210.

21. Chen HY, Cho WC, Sze SC, Tong Y. Treatment of menopausal symptoms with Er-xian decoction: a systematic review. Am J Chin Med. 2008; 36(2):233-244

22. Zhong LL, Tong Y, Tang GW, et al. A randomized, double-blind, controlled trial of a Chinese herbal formula (Er-Xian decoction) for menopausal symptoms in Hong Kong perimenopausal women. Menopause. 2013;20(7):767-776.

23. Higgins JPT, Green S, editors. Cochrane Handbook for Systematic Reviews of Interventions, Version 5.1.0. The Cochrane Collaboration; 2009.

24. Zhang W, Wang S, Zhang R, et al. Evidence of Chinese herbal medicine Duhuo Jisheng decoction for knee osteoarthritis: a systematic review of randomised clinical trials. BMJ Open. 2016;6(1):e008973.
25. Wu JZ, Zhou XM, Zheng CX. The clinical observation of Erxian decoction for primary osteoporosis. Xin Zhong Yi. 2010;42(2):25-26.

26. Zhu QA, Gu MQ. Efficacy of salmon calcitonin plus erxian decoction (EXD) for osteoporosis in postmenopausal women: a clinical observation. Zhong Hua Zhong Yi Yao Xue Kan. 2012;42(2):2806-2809.

27. Gao $C$. The clinical observation of erxian decoction for primary osteoporosis. Hai Xia Yao Xue. 2012;24(7):103-104.

28. Liu YN. The clinical observation of modified erxian decoction for postmenopausal osteoporosis. Zhong Guo Zhong Xi Yi Ji Chu Yi Xue Za Zhi. 2012;18(8):886, 899.

29. Jiang H, Le X, Liu DL, et al. Thirty-five cases efficacy observation of modified erxian decoction for postmenopausal osteoporosis. Zhong Guo Yao Fang. 2014;25(17):2569-2571.

30. Liu $\mathrm{H}$. The efficacy observation of erxian decoction for primary osteoporosis. Dang Dai Yi Xue. 2014;12(7):44-45.

31. Xu XL, Ma X, Sun JJ. The clinical observation and influence on $E_{2}$, ALP of modified erxian decoction in the treatment of postmenopausal osteoporosis. Zhong Guo Zhong Yi Yao Ke Ji. 2016;23(1):68-74.

32. Ye BL, Li SH, Li SL, et al. Clinical study on erxian decoction combined with elcatonin in the treatment of postmenopausal osteoporosis. Zhong Guo Zhong Yi Yao Xin Xi Za Zhi. 2016;23(6):23-26.

33. An J, Yang H, Zhang Q, et al. Natural products for treatment of osteoporosis: the effects and mechanisms on promoting osteoblast-mediated bone formation. Life Sci. 2016;147:46-58.

34. Guo Y, Li Y, Xue L, et al. Salvia miltiorrhiza: an ancient Chinese herbal medicine as a source for anti-osteoporotic drugs. J Ethnopharmacol. 2014;155(3):1401-1416.

35. Liu Y, Liu JP, Xia Y. Chinese herbal medicines for treating osteoporosis. Cochrane Database Syst Rev. 2014;(3):CD005467.

36. Shu B, Shi Q, Wang YJ. Shen (Kidney)-tonifying principle for primary osteoporosis: to treat both the disease and the Chinese medicine syndrome. Chin J Integr Med. 2015;21(9):656-661.

37. Ren YB, Huang JH, Cai WJ, Shen ZY. Shen-Jing as a Chinese medicine concept might be a counterpart of stem cells in regenerative medicine. Chin J Integr Med. Epub 2015 Jul 4.

38. Lin L, Wu S, Tang J. [Clinical observation and experimental study of the treatment of aplastic anemia by warming and tonifying the spleen and kidney]. Zhong Xi Yi Jie He Za Zhi. 1990;10(5):272-274, 259. Chinese.

39. Wu J. [Placebo control and clinical trial of Chinese medicine]. Zhong Xi Yi Jie He Xие Bao. 2010;8(10):906-910. Chinese.

40. Idolazzi L, Fassio A, Gatti D, et al. Duration of treatment for osteoporosis. Reumatismo. 2013;65(1):22-35.

41. Adler RA. Duration of anti-resorptive therapy for osteoporosis. Endocrine. 2016;51(2):222-224.

42. Wang ZQ, Li JL, Sun YL, et al. Chinese herbal medicine for osteoporosis: a systematic review of randomized controlled trails. Evid Based Complement Alternat Med. 2013;2013:356260.

43. Wei X, Xu A, Yin Y, Zhang R. The potential effect of Wuqinxi exercise for primary osteoporosis: a systematic review and meta-analysis. Maturitas. 2015;82(4):346-354.

44. He J, Du L, Liu G, et al. Quality assessment of reporting of randomization, allocation concealment, and blinding in traditional Chinese medicine RCTs: a review of 3159 RCTs identified from 260 systematic reviews. Trials. 2011;12:122.
Clinical Interventions in Aging

\section{Publish your work in this journal}

Clinical Interventions in Aging is an international, peer-reviewed journal focusing on evidence-based reports on the value or lack thereof of treatments intended to prevent or delay the onset of maladaptive correlates of aging in human beings. This journal is indexed on PubMed Central, MedLine,

\section{Dovepress}

CAS, Scopus and the Elsevier Bibliographic databases. The manuscript management system is completely online and includes a very quick and fair peer-review system, which is all easy to use. Visit http://www.dovepress. com/testimonials.php to read real quotes from published authors. 